\title{
USING AHP FOR DECISION MAKING UNDER UNCERTAINTY
}

\author{
Robert F. Bordley ${ }^{1}$ \\ General Motors Corporation \\ Luis G. Vargas \\ University of Pittsburgh
}

\begin{abstract}
This paper develops an axiomatization of the Analytic Hierarchy Process for decision-making under uncertainty. We find that with this axiomatization the AHP priority function is proportional to an exponential of a Dyer-Sarin comparative utility function with a fixed reference point.
\end{abstract}

\section{Introduction}

The analytic hierarchy process(AHP) assesses a ratio scaled priority function $V(a)$ with $V(a)>V(b)$ implying that an individual prefers $a$ to $b$ (and vice versa.). Saaty (1986) and Vargas (1987) developed an axiomatization implying the existence of such value functions for decision-making under certainty. Since uncertainty, however, pervades many choice problems, we propose an axiomatization for priority functions for decision making under uncertainty. We assume that each decision has associated with it a set of consequences, and that each consequence has a likelihood of occurrence that can be measured with probabilities, i.e., the decisions are lotteries or gambles. To develop this axiomatization we extend the axiomatization due to Sarin (1982). Sarin assumed that individuals could assess how much they prefer one gamble to another. Let $\left(d^{\prime}, d^{2}\right)$ denote a pair of gambles $d^{\prime}$ and $d^{2}$. Sarin defined a preference ordering over pairs of gambles with $\left(d^{\prime}, d^{2}\right)$ being ranked higher than $\left(d^{3}, d^{\prime}\right)$ if and only if the individual prefers gamble $d^{t}$ to gamble $d^{2}$ more than he prefers gamble $d^{3}$ to gamble $d^{2}$. After assuming that this ranking of pairs of gambles satisfies the consistency axioms of expected utility theory, Sarin inferred the existence of a ratio-scaled comparative utility function $U$ such that $U\left(d^{l}, d^{2}\right)>U\left(d^{3}, d^{\prime}\right)$ if and only if $\left(d^{l}, d^{2}\right)$ ranks higher than $\left(d^{3}, d^{\prime}\right)$. In addition, if gamble $d^{l}$ gives a payoff of $d_{i}^{1}$ in state $i$, then there exists

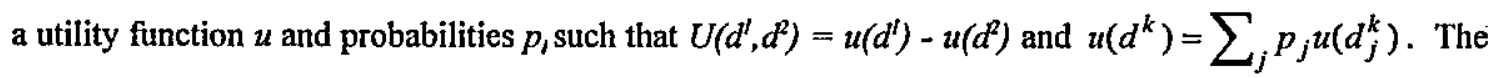
utility function $u\left(d^{*}\right)$ corresponds to the standard utility function of ordinary expected utility theory and it belongs to an interval scale. Thus, for a given individual, any other utility function $u^{*}\left(d^{*}\right)$ must be related to the utility function $u\left(d^{*}\right)$ by a linear transformation $u^{*}=\alpha+\beta u$ with $\beta>0$, and hence, one could model an individual's preferences with $u^{*}$ instead of $u$.

Sarin's result presumed that individuals can compare the absolute differences in value between gambles. However, let us assume that instead of comparing absolute differences in value, individuals could compare relative differences in value. In other words, we can ask an individual to think about the percentage change in value associated with moving from $d^{2}$ to $d^{l}$ versus the percentage change in value associated with changing from $d^{\prime}$ to $d^{3}$. This kind of quantity will, of course, not always be meaningful. In this case, Sarin's argument implies the existence of a comparative value function, $V\left(d^{\prime}, d^{2}\right)$ and $\log$ interval scaled value functions $v\left(d^{*}\right)$ such that the individual considers the relative difference in value

1 The authors thank Vince Barabba, General Manager of the General Motors Corporate Strategy and Knowledge Development Staff, for requesting a comparison of AHP and decision analysis. We also thank Thomas L. Saaty for suggesting our collaboration as well as providing constant encouragement and support. 
between $d^{t}$ and $d^{2}$ greater than the relative difference between $d^{3}$ and $d^{t}$ if and only if $V\left(d^{t}, d^{2}\right)>V\left(d^{3}, d^{t}\right)$ with

$$
V\left(d^{\mathrm{t}}, d^{2}\right)=\frac{v\left(d^{\mathrm{j}}\right)}{v\left(d^{2}\right)} \text { and } \ln \left\{v\left(d^{k}\right)\right\}=\sum_{j} p_{j} \ln \left\{v\left(d_{j}^{k}\right)\right\} .
$$

Now the absolute ordering and the comparative ordering will be equivalent if $U\left(d^{l}, d^{2}\right)=\ln V\left(d^{l}, d^{2}\right)$ and $u\left(d^{*}\right)=\ln v\left(d^{*}\right)$. In this case, the comparative order is redundant and there are no ratio-scaled priority functions. However, there are a wide variety. of cases in which the orderings are distinct. For example, the absolute difference between 15 and 10 exceeds the absolute difference between 6 and 3 but the relative difference between 15 and 10 is less than the relative difference between 6 and 3 . When this happens, Krantz et al. (1971) establish the existence of a ratio-scaled priority function $v\left(d^{*}\right)$ such that $V\left(d^{1}, d^{2}\right)=\frac{v\left(d^{1}\right)}{v\left(d^{2}\right)}$ and $U\left(d^{l}, d^{2}\right)=v\left(d^{\prime}\right)-v\left(d^{2}\right)$.

If there exists a ratio-scaled value function, $v\left(d^{*}\right)$, then we can define it by specifying $V\left(d^{h}, d^{*}\right)$ along with a reference point $d^{o}$ such that $v\left(d^{*}\right)=V\left(d^{*}, d^{o}\right)$. In other words, all the assessments are made relative to a reference point $d^{D}$. As Lebesque (1914) noted:

"It would seem that the principle of economy would always require that we evaluate ratios directly and not as ratios of measurements. However, in practice, all lengths are measured in meters, all angles in degrees, etc.; that is, we employ auxiliary units and, as it seems, with only the disadvantage of having two measurements to make instead of one. Sometimes, this is because of experimental difficulties or impossibilities that prevent the direct comparison of lengths or angles."

Unfortunately, we do not know the reference point. Nonetheless, if we assume that a subject's assessments are more consistent when made relative to the reference point, then we could potentially

- have a subject assess $V\left(d^{\prime}, d^{\prime}\right)$ for all possible combinations of gambles $\left(d^{\prime}, d^{\prime}\right)$,

- identify inconsistencies among the assessments, and

- estimate the priority function as a weighted average of the assessments, discounting those assessments that are inconsistent.

Many different methods have been proposed for constructing such a weighted average. Saaty (1994), of course, proposes computing the weighted average implicitly using an eigenvector approach. Barzilai(1997) and others have proposed using geometric averages. This axiomatization highlights the connection between priority functions and utility theory. The AHP is generally used to assess priority functions by having individuals compare the relative difference in value between two items to either the relative difference in value between two numbers, the relative difference in value between two lines or some other ratio-scaled reference yardstick. This way of assessing intensities, called cross-modality matching, was pioneered by Stevens. As the Appendix in the full version of this paper shows, this procedure also leads to a ratio-scaled priority function if subjects consistently order relative differences in value between alternatives along with relative differences in length for various reference yardsticks.

\section{The Ratio-Scaled Priority Function}

\section{Absolute And Relative Differences}

Let $\boldsymbol{D}$ be the space of gambles $d$, and let $\geq_{0}$ be a binary relation defined on the product space $D \times D=$ $\left\{d^{d} d \mid d^{d}\right.$ is preferred over $\left.d\right\}$, such that $d^{1} d^{2} \geq_{0} d^{3} d^{4}$ if and only if a subject prefers exchanging gamble $d^{2}$ for gamble $d^{\prime}$ more than exchanging gamble $d^{4}$ for gamble $d^{3}$. Let $\geq_{1}$ also be a binary relation defined on $D \times D$ such that $d^{1} d^{2} \geq_{1} d^{3} d^{4}$ if and only if the relative improvement in exchanging gamble $d^{2}$ for gamble $d^{\prime}$ is greater than the relative improvement in exchanging gamble $d^{\prime}$ for gamble $d^{3}$. Let $z_{R}$ be the binary relation $z_{0}$ if $R=0$ and $z_{1}$ if $R=1$. Let $=_{R}$ be a binary relation such that 
$d^{1} d^{2}={ }_{R} d^{3} d^{4}$ if and only if $d^{1} d^{2} \geq_{R} d^{3} d^{4}$ and $d^{3} d^{4} \geq_{R} d^{1} d^{2}$; and let $\neq_{R}$ be a binary relation such that $d^{1} d^{2} \neq_{R} d^{3} d^{4}$ if and only if $d^{1} d^{2} \geq_{R} d^{3} d^{4}$ or $d^{3} d^{4} \geq_{R} d^{1} d^{2}$ and $\neg d^{1} d^{2}=_{R} d^{3} d^{4}$. Likewise, the binary relation $>_{R}$ is defined as $d^{1} d^{2}>_{R} d^{3} d^{4}$ if and only if $d^{1} d^{2} \geq_{R} d^{3} d^{4}$ and $\neg d^{1} d^{2}=_{R} d^{3} d^{4}$. The binary relation $\geq_{R}$ satisfies the following axioms:

A1(R). (Weak Ordering): All improvements can be ordered by $\geq_{R}$ and the order is transitive.

A2(R). (Reciprocity): For all $d^{1} d^{2}, d^{3} d^{4} \in \mathrm{D} \times \mathrm{D}, d^{1} d^{2} \geq_{R} d^{3} d^{4}$ implies $d^{4} d^{3} \geq_{R} d^{2} d^{1}$.

(This axiom is analogous to the AHP axiom postulating that $P\left(d^{1}, d^{2}\right) P\left(d^{2}, d^{1}\right)=1$, where $P\left(d^{i}, d^{j}\right)$ represents the relative preference of $d$ over $d$. Thus, if $P\left(d^{1}, d^{2}\right)>P\left(d^{3}, d^{4}\right)$ then $P\left(d^{4}, d^{3}\right)>P\left(d^{2}, d^{1}\right)$. Since we have not yet proven the existence of such a function $P$, our axiomatization cannot invoke it.)

A3(R). (Internal Transitivity): For all $d^{1}, d^{2}, d^{3}, d^{4}, d^{5}, d^{6} \in D, d^{\mathrm{I}} d^{2} \geq_{R} d^{3} d^{4}$ and $d^{2} d^{5} \geq_{R} d^{4} d^{6}$ implies $d^{1} d^{5} \geq_{R} d^{3} d^{6}$.

We also assume that the set of decisions is rich enough to satisfy:

A4(R). (Restricted Solvability): If $d^{1} d^{2} \geq_{R} d^{3} d^{4} \geq_{R} d^{2} d^{2}$, there exists $d^{6}$ and $d^{7}$ for which $d^{1} d^{6}={ }_{R} d^{3} d^{4}={ }_{R} d^{7} d^{2}$.

We now define a standard sequence of decisions $d^{1}, d^{2}, \ldots, d^{k}, \ldots$ as a sequence of decisions such that each successive decision provides the same amount of relative improvement over its predecessor, i.e., $d^{k} d^{k+1}={ }_{R} d^{k+1} d^{k+2}$.

A5(R). (Finiteness): Let $d^{1}, d^{2}, \ldots, d^{k}, \ldots$ be a standard sequence. If there exist decisions $d^{\prime}$ and $d^{\prime \prime}$ such that $d^{\prime} d^{\prime \prime} \geq_{R} d^{i} d^{1} \geq_{R} d^{\prime \prime} d^{\prime}$ for all $d^{\prime}$, and $d^{i} d^{1} \neq_{R} d^{1} d^{2}, i>2$, then the sequence $d^{1}, d^{2}, \ldots, d^{k}, \ldots$ is finite.

Each of these axioms is applied to $\geq_{0}$ independently of $\geq_{1}$. To interrelate the orderings we need to specify three further axioms.

A1(01). (Trivial Case Ordering Agreement): $d^{2} d^{1}>_{0} d^{1} d^{1}$ if and only if $d^{2} d^{1}>_{1} d^{1} d^{1}$.

A2(01). (Scaling):

1. $d^{\mathrm{I}} d^{2} \geq_{1} d^{\mathrm{l}} d^{1}$ if and only if $d^{1} d^{2} \geq_{0} d^{1} d^{1}$

2. $d^{1} d^{2}=_{R} d^{3} d^{4}={ }_{R} d^{5} d^{6}$ implies $d^{1} d^{3} \geq_{0} d^{3} d^{5}$ if and only if $d^{2} d^{4} \geq_{0} d^{4} d^{6}$.

A3(01). (Non-Identical Orders): There exist some decisions $d^{1}, d^{2}, d^{3}, d^{4} \in D$ for which $d^{1} d^{2}>_{0} d^{3} d^{4}$ but $d^{3} d^{4}>_{1} d^{1} d^{2}$.

Given these conditions on both orderings, Krantz et al. (197i, p.154) proved the following theorem.

Theorem 1: Given a set of decisions $D$ with orderings $\geq_{R}, R=0,1$ that satisfy axioms $A I(R)-A 5(R)$ and $A 1(01)-A 3(01)$, there exist a function $\varphi$ such that for all $d^{1}, d^{2}, d^{3}, d^{4} \in D$

$$
\begin{aligned}
& d^{1} d^{2} \geq_{0} d^{3} d^{4} \Rightarrow \varphi\left(d^{1}\right)-\varphi\left(d^{2}\right) \geq \varphi\left(d^{3}\right)-\varphi\left(d^{4}\right) \\
& d^{1} d^{2} \geq_{1} d^{3} d^{4} \Rightarrow \frac{\varphi\left(d^{1}\right)}{\varphi\left(d^{2}\right)} \geq \frac{\varphi\left(d^{3}\right)}{\varphi\left(d^{4}\right)}
\end{aligned} .
$$




\section{Axioms for Decision-Making Under Uncertainty}

Let $d_{s}^{j}$ be the payoff from decision $\mathrm{j}$ in state $s(s=1,2, \ldots, \mathrm{n})$. When an individual states that $d^{1} d^{2} \geq_{R} d^{3} d^{4}$ she is comparing the improvement of exchanging $d_{s}^{2}$ for $d_{s}^{1}$ with the improvement of exchanging $d_{s}^{4}$ for $d_{s}^{3}$ for each state s. Hence, can write $d^{1} d^{2} z_{R} d^{3} d^{4}$ as:

$$
\left(d_{1}^{1} d_{1}^{2}, \ldots, d_{s}^{1} d_{s}^{2}, \ldots, d_{n}^{1} d_{n}^{2}\right) \geq_{R}\left(d_{1}^{3} d_{1}^{4}, \ldots, d_{s}^{3} d_{s}^{4}, \ldots, d_{n}^{3} d_{n}^{4}\right) .
$$

In utility theory, when an individual compares two situations, the only issues compared are those in which they differ. Thus, we have:

U1(R). (Independence): Let $d^{1} d^{2} \geq_{R} d^{3} d^{4}$ and $d_{i_{j}}^{1} d_{i_{j}}^{2}={ }_{R} d_{i_{j}}^{3} d_{i_{j}}^{4}, \mathrm{j}=1,2, \ldots, \mathrm{n}$. If we change $d^{1} d^{2}$ and $d^{3} d^{4}$ by changing $d_{i j}^{1} d_{i_{j}}^{2}$ and $d_{i_{j}}^{3} d_{i j}^{4}$ to $d_{i j}^{5} d_{i_{j}}^{6}$, still yields $d^{1} d^{2} \geq_{R} d^{3} d^{4}$.

Given this axioms we can introduce a conditional ordering, $\geq_{R_{t}}$, which assumes that the relative improvements in every state but state $i$ are the same.

Definition 1: $d_{i}^{1} d_{i}^{2} \geq_{R_{1}} d_{i}^{3} d_{i}^{4}$ if and only if for any decision $d^{0} \in D$

$$
\left(d_{1}^{0} d_{1}^{0}, \ldots, d_{i-1}^{0} d_{i-1}^{0}, d_{l}^{1} d_{i}^{2}, d_{i+1}^{0} d_{i+1}^{0}, \ldots, d_{n}^{0} d_{n}^{0}\right) \geq_{R}\left(d_{1}^{0} d_{1}^{0}, \ldots, d_{i-1}^{0} d_{i-1}^{0}, d_{i}^{3} d_{i}^{4}, d_{i+1}^{0} d_{i+1}^{0}, \ldots, d_{n}^{0} d_{n}^{0}\right) .
$$

It is clear that if $\geq_{R}$ satisfies $A 1(R)-A 5(R)$, then $\geq_{R_{f}}, \mathrm{i}=1,2, \ldots, \mathrm{n}$, also satisfies $\mathrm{Al}(\mathrm{R})-\mathrm{A} 5(\mathrm{R})$. In addition, we need to assume that there exist decisions whose payoffs are not all equally preferred.

U2(R). (Essential States): There exist decisions $d^{1}, d^{2}, d^{3}, d^{4} \in D$ for which $d_{i}^{1} d_{i}^{2} \neq_{R_{i}} d_{i}^{3} d_{i}^{4}$ for all i.

If there is a state for which this condition fails, then the state can be ignored in the analysis.

U3(R). (Solvability): Let $d^{1}, d^{2} \in D$ and $d_{k}^{1}=d_{k}^{2}$ for all $k \neq i$, and let $d^{1} d^{0} \geq_{R_{1}} d^{2} d^{0}$ for some $d^{0} \in D$. If there exists a decision $d^{3} \in D, d^{1} d^{0} \geq_{R} d^{3} d^{0} \geq_{R} d^{2} d^{0}$, then a decision $d^{4}$ can be constructed which is identical to decision $d^{l}$ except in state i with $d^{4} d^{0}={ }_{R_{t}} d^{3} d^{0}$.

Let $d^{\mathrm{l}}(x, i) \equiv\left(d_{1}^{1}, \ldots, d_{i-1}^{1}, x, d_{i+1}^{1}, \ldots, d_{n}^{1}\right)$ and let $d^{1}(x, i ; y, j) \equiv\left(d_{1}^{1}, \ldots, d_{i-1}^{1}, x, d_{i+1}^{1}, \ldots, d_{j-1}^{\mathrm{1}}, y, d_{j+1}^{\mathrm{l}}, d_{n}^{1}\right)$.

Definition 2: A multi-state standard sequence is a sequence $\left(b_{1}, b_{2}, \ldots, b_{l}, \ldots\right)$ where $d^{1}\left(b_{k}, i ; p, j\right)=d^{1}\left(b_{k+1}, i ; q, j\right)$ with $p \neq q$ for all $\mathrm{i}, \mathrm{j}$ and $\mathrm{k}$.

Definition 3: A standard sequence is strictly bounded if for all $b_{k} \in\left(b_{1}, b_{2}, \ldots, b_{i}, \ldots\right)$ there exist some $\mathbf{x}$ and y such that $d^{1}(x, i) \geq_{R} d^{1}\left(b_{k}, i\right) \geq_{R} d^{1}(y, i)$.

U4(R). (Finiteness): All standard sequences which are strictly bounded are finite.

U5(R). (State Independent Utilities): The value of a consequence of a decision does not depend on the state, i.e.,for all $x, x^{\prime}$ and $x "$

(a) $d^{1}(x, i) d^{2}\left(x^{\prime}, i\right)==_{R} d^{2}\left(x^{\prime}, i\right) d^{3}\left(x^{\prime \prime}, i\right) \Rightarrow d^{1}(x, j) d^{2}\left(x^{\prime}, j\right)={ }_{R} d^{2}\left(x^{\prime}, j\right) d^{3}\left(x^{\prime \prime}, j\right)$

(b) $d^{1}(x, i) d^{0} \geq_{R} d^{2}\left(x^{1}, i\right) d^{0} \Rightarrow d^{1}(x, j) d^{0} \geq_{R} d^{2}\left(x^{\prime}, j\right) d^{0}$

Theorem 2: If the binary relation $\geq_{R}$ satisfies axioms $A l(R), U I(R)-U 4(R)$, and $A 5(R)$, there exists a function $\phi$ such that $d^{1} d^{2} \geq_{R} d^{3} d^{4} \Leftrightarrow \sum_{s \in S} \phi_{s}\left(d_{s}^{1} d_{s}^{2}\right) \geq \sum_{s \in S} \phi_{s}\left(d_{s}^{3} d_{s}^{4}\right)$, where $S$ is the state space. 
The function $\phi$ represents the perceived gain to the decision maker when shifting from one decision to another.

\section{Derivation of Interval and Ratio Scale Utility Functions}

In Theorem 1 we proved that:

$$
d^{1} d^{2} \geq_{0} d^{3} d^{4} \Rightarrow \phi\left(d^{1}\right)-\phi\left(d^{2}\right) \geq \phi\left(d^{3}\right)-\phi\left(d^{4}\right) \text { and } d^{1} d^{2} \geq_{1} d^{3} d^{4} \Rightarrow \frac{\phi\left(d^{1}\right)}{\phi\left(d^{2}\right)} \geq \frac{\phi\left(d^{3}\right)}{\phi\left(d^{4}\right)} \text {. }
$$

Now, the function $\phi$ is multidimensional, $\phi\left(d^{i}\right)=\phi\left(d_{1}^{i}, d_{2}^{i}, \ldots, d_{s}^{i}, \ldots, d_{n}^{i}\right)$ and hence, the results of both theorems will only hold if $\phi$ is separable as a function of the state, i.e., $\phi\left(d^{i}\right)=\sum_{s \in S} v_{s}\left(d_{s}^{i}\right)$. Since axiom $\mathrm{U}$ (R) implies that $v_{s}$ is independent of the state $\mathrm{s}$, then

$$
d^{1} d^{2} z_{0} d^{3} d^{4} \Leftrightarrow \sum_{s \in S}\left\{v\left(d_{s}^{\mathrm{1}}\right)-v\left(d_{s}^{2}\right)\right\} \geq \sum_{s \in \mathcal{S}}\left\{v\left(d_{s}^{3}\right)-v\left(d_{s}^{4}\right)\right\}
$$

or

$$
d^{1} d^{2} \geq_{0} d^{3} d^{4} \Leftrightarrow \sum_{s \in S} v\left(d_{s}^{1}\right)-\sum_{s \in S} v\left(d_{s}^{2}\right) \geq \sum_{s \in S} v\left(d_{s}^{3}\right)-\sum_{s \in S} v\left(d_{s}^{4}\right) .
$$

Similarly, for the binary relation $\geq_{1}$, there must exist a function $w$, for which we have:

$$
d^{1} d^{2} \geq_{1} d^{3} d^{4} \Leftrightarrow \frac{\sum_{s \in S}\left[w\left(d_{s}^{1}\right)\right]}{\sum_{s \in S}\left[w\left(d_{s}^{2}\right)\right]} \geq \frac{\sum_{s \in S}\left[w\left(d_{s}^{3}\right)\right]}{\sum_{s \in S}\left[w\left(d_{s}^{4}\right)\right]} .
$$

If the function $v\left(d_{s}^{i}\right)=v\left(d_{A}^{i}\right)$, for all $s \in A \subset S$, and $p(A)$ represents the fraction of states in $\mathrm{A}$, then we have:

$$
d^{1} d^{2} \geq_{0} d^{3} d^{4} \Leftrightarrow \sum_{A \subset S} p(A) v\left(d_{A}^{1}\right)-\sum_{A \subset S} p(A) v\left(d_{A}^{2}\right) \geq \sum_{A \subset S} p(A) v\left(d_{A}^{3}\right)-\sum_{A \subset S} p(A) v\left(d_{A}^{4}\right)
$$

and

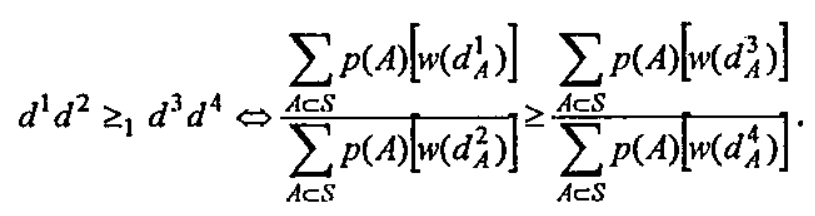

These results can be summarized in the following corollaries.

Corollary 2: If $\geq_{0}$ satisfies axioms $A 1(0)-A 5(0), U 1(0)-U 5(0)$, then

$$
d^{1} d^{2} \geq_{0} d^{3} d^{4} \Rightarrow v\left(d^{1}\right)-v\left(d^{2}\right) \geq v\left(d^{3}\right)-v\left(d^{4}\right)
$$

where $v\left(d^{i}\right)=\sum_{A \subset S} p(A) v\left(d_{A}^{i}\right), i=1,2,3,4$

Corollary 3: If $\geq_{1}$ satisfies axioms $A 1(0)-A 5(0)$, U1(0)-U5(0), and A1(01)-A3(01), then

$$
d^{1} d^{2} \geq_{1} d^{3} d^{4} \Rightarrow \frac{w\left(d^{1}\right)}{w\left(d^{2}\right)} \geq \frac{w\left(d^{3}\right)}{w\left(d^{4}\right)}
$$

and $w$ is unique to within a multiplicative constant. 


\section{AHP's Normative Ratio Scale}

While this establishes the existence of a ratio-scaled priority function, the AHP actually constructs this value function (see Vargas, 1986, for a study of the relationship between utilities and priorities of gambles) by having an individual compare the relative differences in value between gambles with the relative differences in, for example, the length of various yardsticks. This kind of cross-modality matching procedure has been studied by the psychophysicist S. Stevens, who argued that it could lead to ratio scales. To develop an axiomatization for this kind of assessment procedure, we let $\left(d^{1} d^{2}\right)$ denote the comparison of the value of the gamble $d^{3}$ to $d^{2}$ and let $\left(y^{1} y^{2}\right)$ denote a comparison of the length of yardstick $y^{1}$ to the length of the yardstick $y^{2}$. We then define an ordering over the pairs of gambles and the pairs of yardsticks. Assuming axioms like the those assumed previously establishes the existence of a ratio-scale priority function over gambles.

\section{Conclusions}

We have shown that the AHP could be axiomatized by extending Sarin's axiomatization of absolute differences in preference intensity to an axiomatization of both absolute and relative differences in preference intensity. The result establishes the existence of a priority function which is closely related to a comparative utility function where gambles are always being compared to a common reference gamble. Hence assessing the priority function requires both the identification of this reference gamble as well as the comparison of all other gambles to this reference gamble. Saaty's eigenvector method is a way of estimating this fixed-reference comparative utility function, given no a priori knowledge of that fixed reference point. However, estimating the reference gamble from the gambles under consideration means that expanding the number of gambles under consideration could change the reference gamble, and thus change the assessed value of all other gambles.

\section{References}

Barzilay, J., "Deriving Weights from Pairwise Comparison Matrices," Journal of the Operational Research Society 48, 1997.

Krantz, D., D. Luce, P. Suppes and A. Tversky, Fundamentals of Measurement, Vol. 1, Academic Press, New York, 1971.

Lebesgue, H., Lecons sur L'Integration, $2^{\text {nd }}$ Edition, Gauthiers-Villars, Paris, 1928.

Saaty, T.L., "Axiomatic Foundations of the Analytic Hierarchy Process," Management Science 21, 1986.

Saaty, T.L., Fundamentals of Decision Making and Priority Theory, RWS Publications, 1994.

Sarin, R., "Strength of Preference and Risky Choice," Operations Research 30, 5, 1982.

Stevens, S.S., "On the Psychophysical Law," Psychological Review 64: 153-181, 1957.

Vargas, L.G., "Utility Theory and Reciprocal Pairwise Comparisons: The Eigenvector Method," SocioEconomic Planning Sciences 20, 6: 387-391, 1986.

Vargas, L.G., "Priority Theory and Utility Theory," Mathematical Modelling 9, 3-5:381-385, 1987. 